\title{
Bore Disruption: An Unusual Mechanical Failure of Two Hip Hemiarthroplasties
}

\author{
Federico Dettoni ${ }^{1} \quad$ Umberto Cottino $^{1}$ Silvia Spriano \\ ${ }^{1}$ Ospedale Mauriziano Umberto I, SCDU Ortopedia e Traumatologia, \\ Turin, Italy \\ 2 Department of Applied Science and Technology, Politecnico di \\ Torino, Turin, Italy \\ ${ }^{3}$ Department of Orthopaedics and Traumatology, Università degli \\ Studi di Torino, Turin, Italy \\ Joints 2017;5:51-56.
}

\author{
Address for correspondence Federico Dettoni, MD, Ospedale \\ Mauriziano Umberto I, SCDU Ortopedia e Traumatologia, Largo \\ Filippo Turati 62, 10128 Turin, Italy (e-mail: dettonif@gmail.com).
}
Abstract
Keywords
- fatigue
- fracture
- hemiarthroplasty
- hip
- scanning electron
microscopy

We report a case of two hemiarthroplasties (HAs) that underwent an unexpected mechanical failure. Two patients affected by femoral fractures were treated with an HA. At 5 and 7 years after surgery, they showed a breakage of the $\mathrm{HA}$ at the head-stem junction. We analyzed macroscopically and microscopically (by a scanning electron microscopy) the surfaces of the broken prostheses. In both cases, a fracture was detected starting at the joining point between the femoral head and the slot that receives the taper cone of the stem (the so-called bore), with the typical macroscopic and microscopic pattern of a fatigue fracture. The fatigue fracture resulted from repeated cycles of torsional stresses throughout the years. In literature, we did not find any other report of such HA failure.

\section{Introduction}

Hip fractures (HFs) bear a heavy burden on health economics: the annual cost of HF treatment in the United States in 2006 was estimated at nearly $\$ 10$ billion. ${ }^{1}$ As osteoporosis affects rough 10 million Americans, and more than 1.5 million fractures are expected every year. ${ }^{2}$ The treatment of $\mathrm{HF}$ has to be definitive and effective and should avoid the risk of a reintervention: this is fundamental for the frail general conditions of patients who can be too weak to overtake two different surgeries. In case of medial (intracapsular) HF, in the elderly population with low functional demand, we usually implant a hip hemiarthroplasty (HA).

$\mathrm{HA}$ is a very effective treatment, and failure is a rare complication. The most frequent complications after HA are HA dislocation, infection, and aseptic loosening, whereas mechanical failure of the implant (i.e., prosthesis breakage) is extremely rare. In literature, only two papers reported a single case and a case series of prosthesis rupture, respectively. ${ }^{3,4}$ One paper reported an HA head equatorial rupture. ${ }^{3}$ The case series by Grupp et al ${ }^{4}$ described 68 cases of implant breakage at the neck-head junction.

In our institution, we encountered two cases of broken HA head at the head-bore junction, apparently with the same pattern, in two different patients. The aim of this study was to report the two cases and to investigate the causes of failure of the two implants by a scanning electron microscopy (SEM) analysis of the retrieved pieces.

\section{Case Presentation}

\section{Case 1}

First case was of a 67-year-old woman affected by hypertension and a non-Hodgkin lymphoma, who was referred to our observation in 2008. She was plainly active at the time of trauma. The patient was affected by a femoral neck fracture, secondary to a lymphoma localization. An Ellittica (Samo, Bologna, Italy) cemented HA was implanted (47-mm head, $M$ stem). After surgery, the patient returned to her normal life. She came back to our observation 5 years later, in 2013, for an atraumatic hip pain at the same hip. She was first evaluated in the emergency department, where a plain radiograph was performed. At a first sight, the diagnosis was not clear, and a computed tomography (CT) scan was requested. Observing the $\mathrm{CT}$ images, a dislocation inside the head of the neck and Morse taper of the stem was detected, and a prosthesis failure was diagnosed.

The HA was revised to a total hip arthroplasty (Wagner SL Stem with Trilogy cup, Zimmer Inc., Warsaw, Indiana, United States) (- Fig. 1). After surgery, the patient returned 

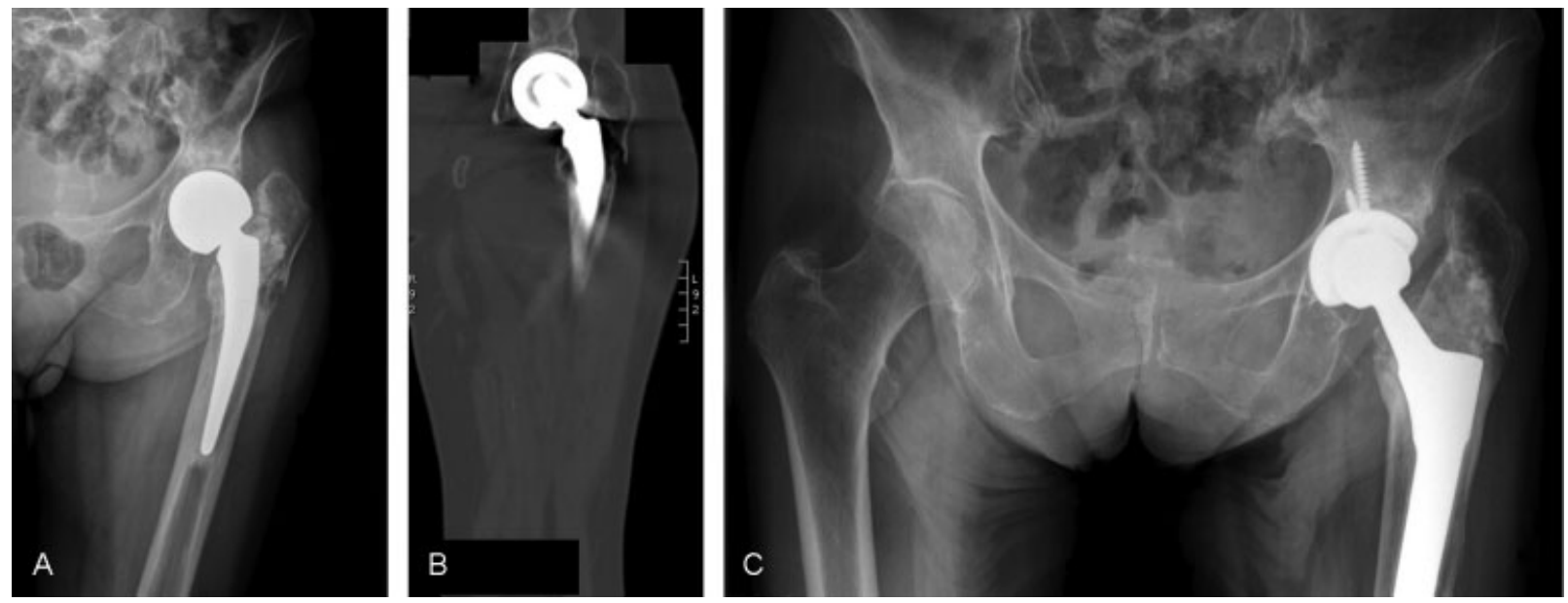

Fig. 1 Case 1. (A) Preoperative X-ray and (B) computed tomography scan of broken hemiarthroplasty. (C) Postoperative X-ray of conversion to total hip arthroplasty.

home and came back to pain-free deambulation, but 1 year later died due to lymphoma complications.

\section{Case 2}

The second case was of an 80-year-old man affected by atrial fibrillation, non-Hodgkin lymphoma, and chronic renal insufficiency. He referred to our emergency department for a femoral neck fracture in 2003. Prior to the fracture, the patient was active, with complete deambulatory autonomy, and reported jogging twice a week. An Ellittica-cemented HA (Samo, Bologna, Italy) was implanted (51-mm head, XL stem). After surgery, the patient returned to his normal life, including sports. In 2010, he felt a sudden pain in the hip while walking and then referred to our emergency department. A plain radiograph was performed. A shortening of the stem neck was clearly detected; therefore, a CT scan was requested. The migration of the neck of the HA inside the head was clearly visible, and a breakage of the head cone was diagnosed.
The HA was converted to a total hip arthroplasty (THA), implanting an ABG cup (Stryker, Kalamazoo, Michigan, United States) (56 $\mathrm{mm}$ ) with a polyethylene inlay, leaving the implanted stem and replacing the broken head with a ceramic head (Samo, Bologna, Italy) (-Fig. 2).

\section{Scanning Electron Microscopy Analysis}

Both HA heads were sent for an analysis to the Department of Applied Science and Technology of Politecnico di Torino. The findings of macro-optical observations and scanning electron microscope (SEM-FEI, Quanta Inspect 200, FEI) analysis are reported in the following.

Both retrieved broken HA heads were chromium cobalt alloy $(\mathrm{CrCo})$ heads, with a design of a metallic sphere with an empty core. In both cases, at macroscopic inspection, the slot that houses the cone taper of the stem (the so-called bore) was detached and dislocated inside the head.

The fracture surfaces of case 1 were two annuli, with an inner diameter of $8 \mathrm{~mm}$ and an external diameter of $12 \mathrm{~mm}$.
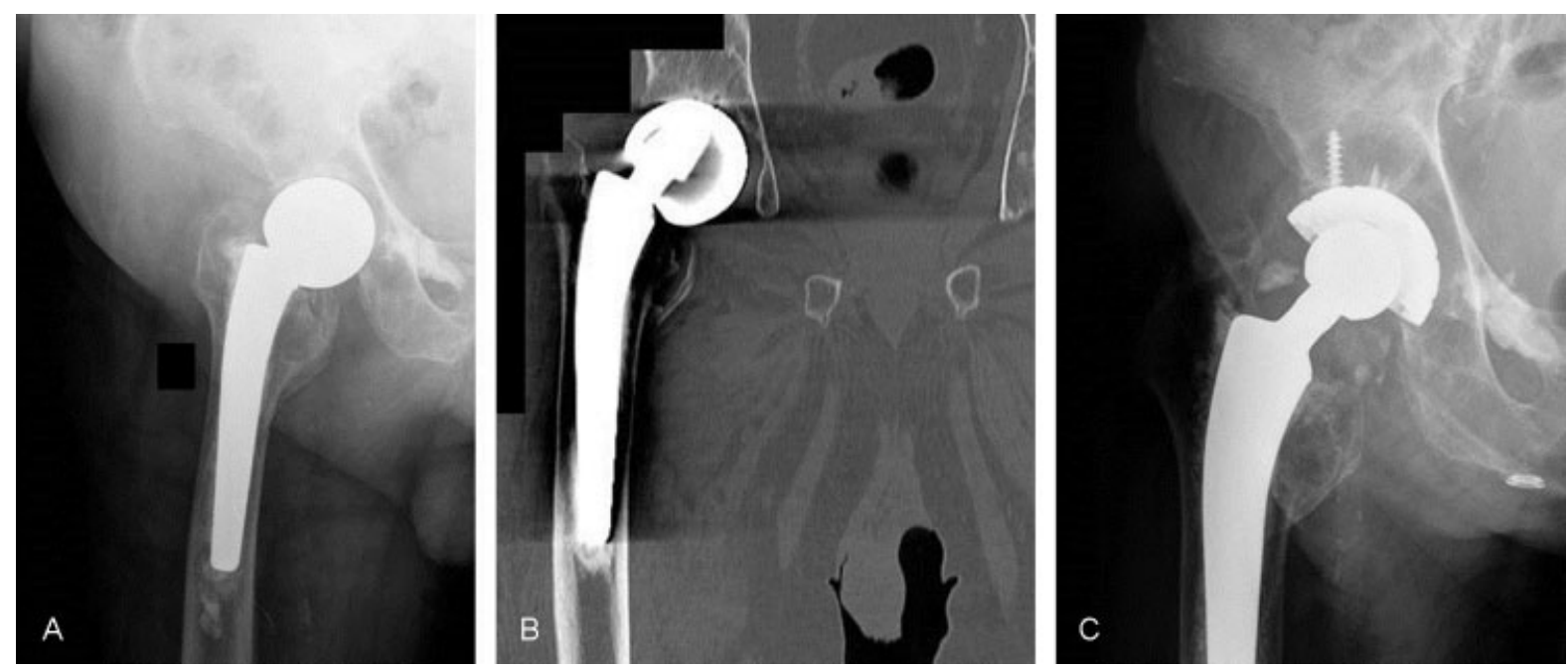

Fig. 2 Case 2. (A) Preoperative X-ray and (B) computed tomography scan of broken hemiarthroplasty. (C) Postoperative X-ray of conversion to total hip arthroplasty. 
It was possible to observe only a single site of initiation of the fatigue fracture, with no morphological or microstructural defect near the initiation site. More than half of the fracture surface was due to a mechanism of fatigue, whereas the other half corresponded to final fracture. The mechanism of fracture observed was the so-called transgranular and not intergranular pattern. Striations appeared as relatively evenly spaced parallel lines; this pattern of fracture is often present on fatigue fracture surfaces of cobalt alloys. Each striation actually represents a shallow crack resulting from a single stress cycle of approximately 5 microns. Some zones with evidences of final fracture were observed only near the outer edge of the fracture surfaces with the presence of a lot of cleavage planes, in different directions on the different crystals, suggesting that final fracture occurs with a brittle mechanism. To check the chemical composition of the metal alloy of the prosthesis, an energy dispersive spectroscopy (EDS) analysis was performed: cobalt, chromium, and molybdenum have been identified in the ratios required for the cobalt-alloy 66Co-28Cr-6Mo (ASTM F 1537), declared by the manufacturer, without any trace of contamination (-Fig. 3 ).

In case 2 , the mechanism of fracture was transgranular, similarly to that noticed in case 1 . Unlikely case 1 , two initiation points of the fracture were identified, located on the outer edge of the samples, at approximately 180 degrees one from the other. The second initiation point (the most interesting) was identified in correspondence of an evident cavity in the fracture surface of the femoral head and in a protuberance of the fracture surface of the head cone. Moving radially from the outer edge (first initiation point) toward the center of the prosthesis, the propagation appeared initially slow and then increasingly faster until the inner edge, where the final part of the fracture occurred, with wrinkled and uneven structure (brittle fracture). The difference observed between the two cases was a widespread presence of fatigue striations in multiple directions in case 2 , according to the presence of two crack initiation points. Observing the second initiation point at a low magnification, a macroscopic lateral groove was noted. This groove came from mechanical processing by a tool, as shown by the regular streaks on its inner surface. Around this initiation, the fatigue fracture propagation was quite limited and evolved rapidly into final fracture. It can be concluded that the defect, resulting from machining, was a stress concentration factor, critical for the initiation and propagation of the fracture. Even in this case, the EDS chemical analysis did not record either contamination of relief or significant chemical differences in respect to the declared chemical composition (-Fig. 4).

\section{Discussion}

The joining point between the femoral head and the stem seat seemed to be the critical issue of these two HAs, even if the situation worsens when some factors are present (such as machining defects or torsional stress), and different initiation causes can be critical for nucleation of the fracture crack. The fatigue fracture had a wide propagation in both cases, covering about half of the fracture surface. This confirms the good quality of the employed materials, as they are able to sustain the load even if presenting a widely reduced crosssection. Impacts or overloads may have occurred on these
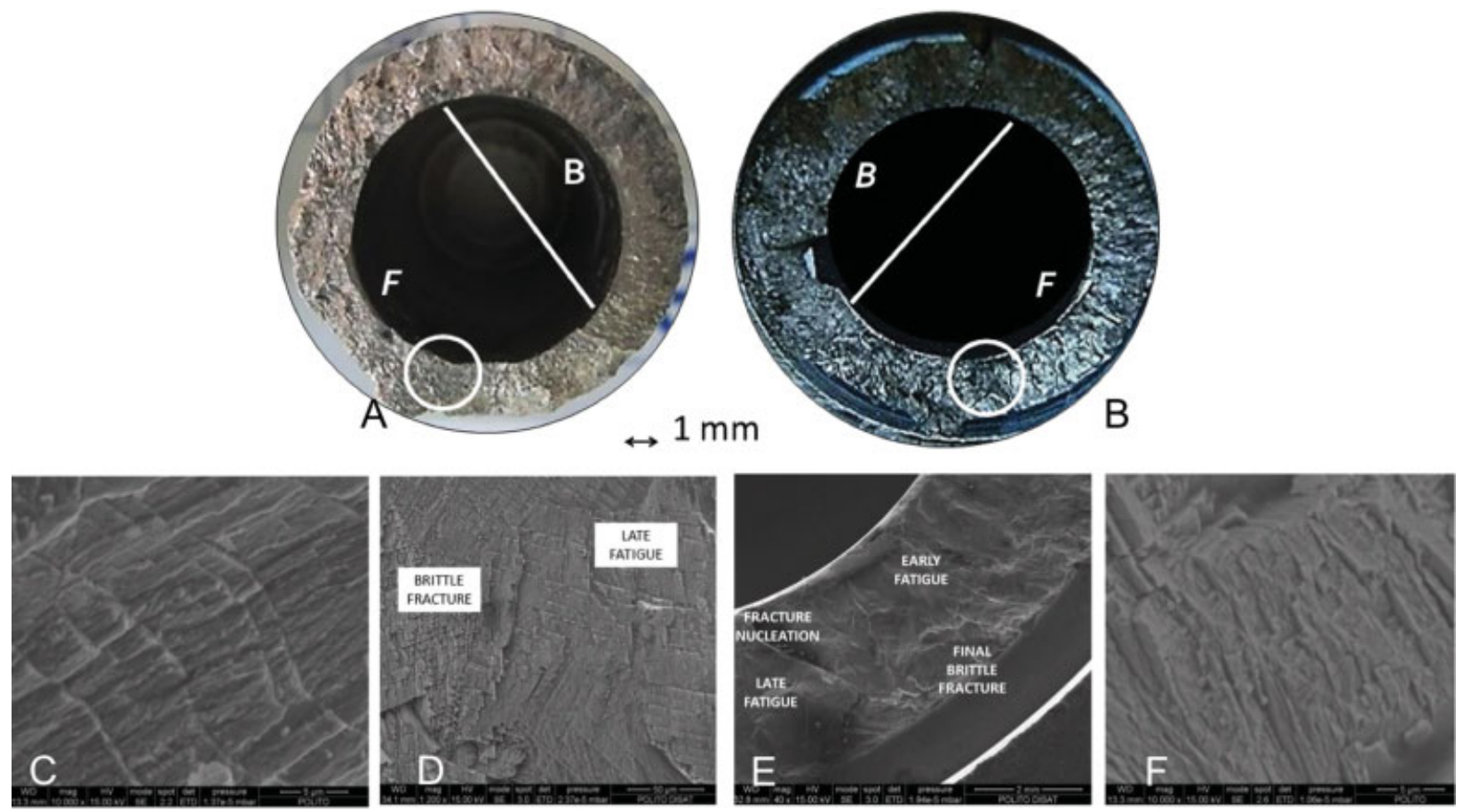

Fig. 3 Scanning electron microscopy analysis of case 1. (A) Bore section. (B) Head side. "F" stands for "fatigue fracture" and "B" stands for "brittle fracture." The circles correspond to the nucleation site of the fracture. (C) A magnification of the late fatigue zone on the bore. (D) A magnification of the transition area between the late fatigue and final brittle zone on the bore. (E) An overview of the nucleation site of the fracture on the head. (F) A magnification of the early fatigue zone on the head. 

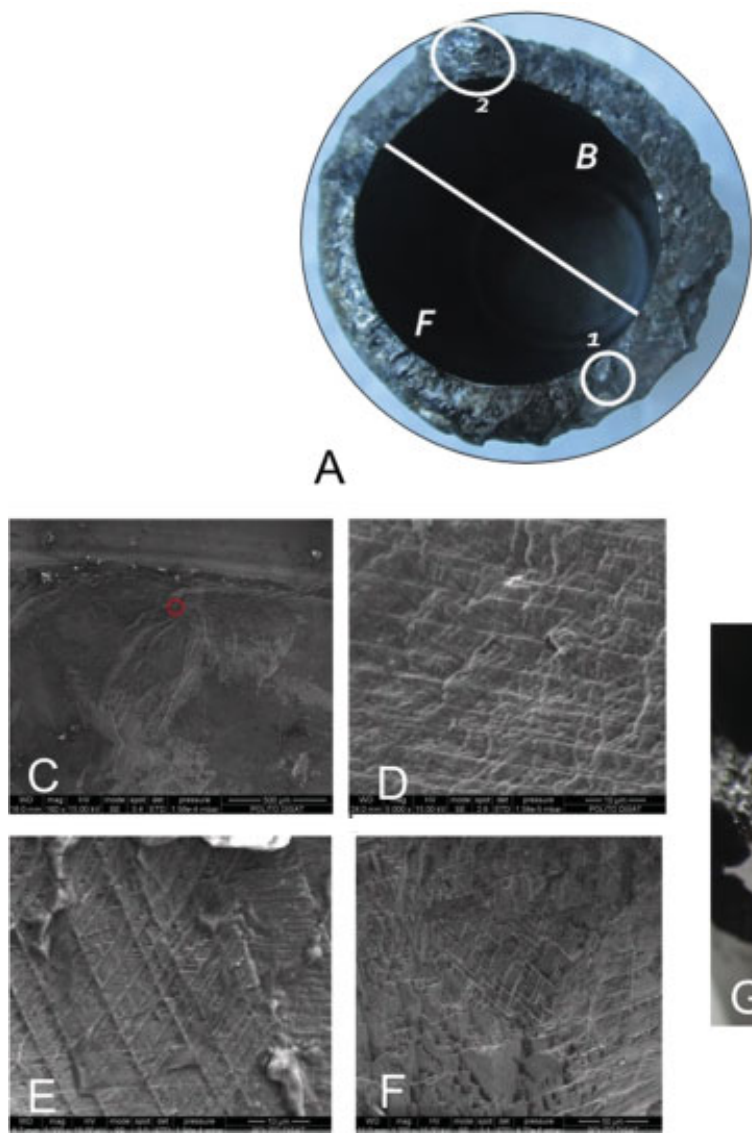

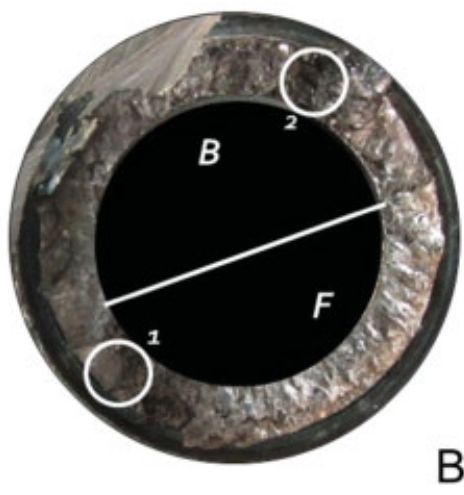

B
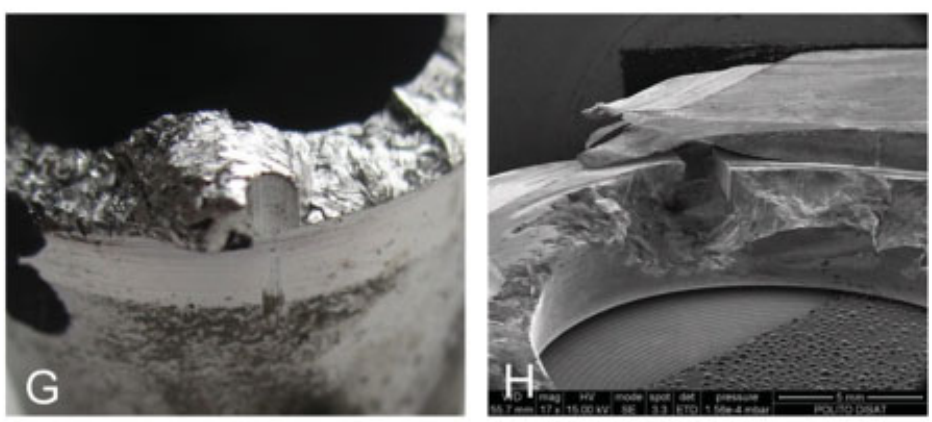

Fig. 4 Scanning electron microscopy analysis of case 2. (A) Bore section. (B) Head side. "F" stands for "fatigue fracture" and "B" stands for "brittle fracture." The circles correspond to the nucleation site of the fracture. (C) An overview of the nucleation site of the fracture on the head. (D) A magnification of early fatigue zone on the head on the head. (E) A magnification of the late fatigue zone with striations in multiple directions. (F) A magnification of the final brittle fracture on the head. $(\mathbf{G}, \mathrm{H})$ macroscopic images of the fracture surfaces of the prosthesis, near point 2 of initiation of the fracture: a later groove can be seen on both the stem seat and the femoral head.

failure cases, as initiation or final events, but the fracture was not a crash event in both the examined cases. The final fracture occurred through brittle mechanism, as expected for cobalt alloys.

Mechanical breakage of prosthesis components is a very rare complication; in these cases, we described a new pattern of failure of an HA head. We analyzed the signs on the prosthesis in the site of breakage to understand the reason for rupture.

In both cases, a fracture was detected starting at the joining point between the femoral head and the stem seat, the so-called bore, with the typical macroscopic and microscopic (SEM) pattern of a fatigue fracture. The fatigue fracture resulted from repeated cycles of torsional stresses, as it is expected to happen in usual daily walking.

It can be stated that the failure of the prosthesis in case 1 has occurred for a high number of fatigue cycles. The appearance of the fracture surface of case 2 is consistent with the presence of a torsional stress and two initiation points. The second initiation point showed a macroscopic lateral groove that originated from mechanical processing by a tool. The defect, resulting from machining, was a stress concentration factor, critical for the initiation and propagation of the fracture. A responsibility of the machining process toward fracture initiation can be therefore hypothesized.

In literature, we found two papers dealing with similar mechanical failures of hip implants. Giori ${ }^{3}$ reported an equatorial fracture of the $\mathrm{CrCo}$ femoral head in a monoblock Anatomic Medullary Locking (DePuy) femoral component of a THA. Grupp et $\mathrm{al}^{4}$ reported 68 titanium modular neck adapters (Metha Short Hip Stem, Aesculap AG, Tuttlingen, Germany) ruptures analyzed using microscopic and chemical methods, and simulated biomechanical failure tests. Both papers showed similar fatigue fracture patterns, even if the factures occurred at different sites.

We can state that the materials of our retrieved HA were of good quality and that they behaved as expected. Exception was made for the second case in which it is possible that a machining defect, leading to a structural weakness, accelerated the normal wearing process. Positioning of the HA was considered good; no impingement or orientation defects were observed during revision surgery.

The only question that can be put on the basis of our analysis is if the HA head design (a metallic sphere with an empty core) could raise stresses at the stem-head junction that could lead to fatigue fractures of the stem seat (the bore). Interestingly, both failures occurred after a long time after 
the operation, in relatively healthy active patients and with large diameter heads. Our suspect is that large diameter heads in active patients created a high stress at the headstem junction that in metallic heads with an empty core may have determined a fatigue fracture, despite the manufacturer using excellent materials and good machining processes. A full head (i.e., a full metal sphere), despite the weight of the materials, would not face this type of failure.

After a complete analysis of these two isolated cases, we could not isolate any sign that can anticipate HA weakness and consequent premature unexpected breakage.

In conclusion, this paper described and analyzed a new mode of mechanical failure of HA implants, never described in literature earlier. The disruption at the bore-head junction was determined in both cases by a fatigue fracture caused by repeated high torsional stresses throughout the years, despite the manufacturer using excellent materials and good machining processes.

\section{References}

1 Raaymakers EL. Fractures of the femoral neck: a review and personal statement. Acta Chir Orthop Traumatol Cech 2006; 73(01):45-59

2 Becker DJ, Kilgore ML, Morrisey MA. The societal burden of osteoporosis. Curr Rheumatol Rep 2010;12(03):186-191

3 Giori NJ. Unexpected finding of a fractured metal prosthetic femoral head in a nonmodular implant during revision total hip arthroplasty. J Arthroplasty 2010;25(04):659.e13-659.e15

4 Grupp TM, Weik T, Bloemer W, Knaebel HP. Modular titanium alloy neck adapter failures in hip replacement-failure mode analysis and influence of implant material. BMC Musculoskelet Disord 2010;11:3. Doi: 10.1186/1471-2474-11-3 